\title{
Isotretinoin Use and the Risk of Inflammatory Bowel Disease: A Case Control Study
}

\author{
Seth D. Crockett, MD ${ }^{1}$, Carol Q. Porter, BS ${ }^{3}$, Christopher F. Martin, MSPH ${ }^{1}$, Robert S. \\ Sandler, MD, MPH ${ }^{1}$, and Michael D. Kappelman, MD, MPH $^{2}$ \\ ${ }^{1}$ Division of Gastroenterology and Hepatology, Department of Medicine, University of North \\ Carolina at Chapel Hill, Chapel Hill, NC 27599 USA \\ 2 Division of Gastroenterology, Department of Pediatrics, University of North Carolina at Chapel \\ Hill, Chapel Hill, NC 27599 USA \\ ${ }^{3}$ Cecil G. Sheps Center for Health Services Research, University of North Carolina at Chapel Hill, \\ Chapel Hill, NC 27599 USA
}

\begin{abstract}
Introduction-Isotretinoin is commonly prescribed for the treatment of severe acne. Though cases of inflammatory bowel disease (IBD) have been reported in isotretinoin users, a causal association remains unproven.
\end{abstract}

Methods-We performed a case-control study using a large insurance claims database. Incident cases of IBD were identified and matched to 3 controls on the basis of age, gender, geographical region, health plan, and length of enrollment. Isotretinoin exposure was assessed in a 12 month period prior to case ascertainment. Conditional logistic regression was used to adjust for the matching variables.

Results-The study population contained 8,189 cases (3,664 CD and 4,428 UC) and 21,832 controls. Sixty subjects (24 cases and 36 controls) were exposed to isotretinoin. Ulcerative colitis was strongly associated with prior isotretinoin exposure (OR 4.36, 95\% CI 1.97, 9.66). However, there was no apparent association between isotretinoin and Crohn's disease (OR 0.68, 95\% CI $0.28,1.68$ ). Increasing dose of isotretinoin was associated with elevated risk of UC (OR per $20 \mathrm{mg}$

Corresponding author: Seth D. Crockett, Department of Medicine, Division of Gastroenterology and Hepatology University of North Carolina School of Medicine, CB\#7080, Chapel Hill, NC 27599-7080, seth_crockett@ med.unc.edu, Phone: (919) 966-2514, Fax: (919) 843-2508.

Guarantor of submission: Seth D. Crockett

Disclosures:

Seth Crockett: None

Carol Porter: None

Christopher Martin: None

Robert Sandler: Serves as a consultant to Pozen, Inc.

Michael Kappelman: None

Authorship:

Seth Crockett: Participated in study concept and design, performed statistical analysis and interpretation of data, drafted the initial manuscript and revised it for important intellectual content.

Carol Porter: Contributed technical and computer programming support and performed data extraction from administrative claims database.

Christopher Martin: Advised on study design and performance, and data analysis. Critically revised manuscript for important intellectual content.

Robert Sandler: Advised on study design and performance. Critically revised manuscript for important intellectual content. Michael Kappelman: Conceived and designed project, provided analytical oversight, and critically revised manuscript for important intellectual content. 
increase in dose: $1.50,95 \%$ CI 1.08, 2.09). Compared to non-users, the risk of UC was highest in those exposed to isotretinoin for more than 2 months (OR 5.63, 95\% CI 2.10, 15.03).

Conclusions-Ulcerative colitis but not Crohn's disease is associated with prior isotretinoin exposure. Higher dose of isotretinoin appears to augment this risk. Though the absolute risk of developing UC after taking isotretinoin is likely quite small, clinicians prescribing isotretinoin as well as prospective patients should be aware of this possible association.

\section{Introduction}

Isotretinoin (Accutane ${ }^{\circledR}$, Amnesteem ${ }^{\circledR}$, Sotret ${ }^{\circledR}$, Claravis ${ }^{\circledR}$ ) is a vitamin $A$ analog that was FDA approved for the indication of severe nodulocystic acne in 1982. Subsequent to its approval, isotretinoin has become a widely prescribed medication despite its well-known teratogenic effects (1-4). In the post-marketing period, several reports of patients developing inflammatory bowel disease (IBD) following treatment with isotretinoin were published (511). Inflammatory bowel disease was subsequently listed in the package insert for Accutane as an adverse gastrointestinal effect (12). Additionally, the potential connection between isotretinoin and IBD has spurred a number of multi-million dollar lawsuits (13). In June 2009, Roche Pharmaceuticals pulled Accutane from the US market in the face of competition from generic makers of isotretinoin, who now supply more than $95 \%$ of the US market. The company also cited "high costs from personal-injury lawsuits" as a reason for withdrawal, though they dispute the validity of claims that isotretinoin is related to IBD (14).

Evidence supporting a causal connection between isotretinoin and IBD largely consists of isolated case reports (5-11). In addition, an analysis of the Food and Drug Administration's MedWatch adverse event monitoring system between 1997 and 2002 revealed 83 reported cases of de novo IBD developing in persons exposed to isotretinoin (15). An association between isotretinoin and IBD may be biologically plausible based on the pleotropic effects of isotretinoin on the innate and adaptive intestinal immunoregulatory capacity (16). Nevertheless, while these case reports are certainly a cause for concern, they are insufficient evidence to determine whether isotretinoin users have a risk of IBD that exceeds the background rate (17).

To date, only a single case control study performed in Manitoba has evaluated this topic and did not demonstrate an association between isotretinoin use and subsequent development of IBD (18). However, this study did not include an analysis of temporality, dose-response, and other factors important in determining causality, and may have underestimated the effect size. To further evaluate the possibility of an association between isotretinoin and IBD, we performed a case-control study using a large U.S. administrative claims database.

\section{Methods}

\section{Study Design and Data Source}

We conducted a case-control study using administrative claims data from individuals captured within the PharMetrics Patient-Centric Database (IMS Health, Watertown, MA) from January 1, 2000 to December 31, 2005. This longitudinal, individual-level database includes 87 health plans across 33 states in the US. At the time the data was extracted, the database contained information on approximately 55 million Americans. Data elements used in this analysis included inpatient and outpatient diagnoses (International Classification of Diseases, $9^{\text {th }}$ Revision, Clinical Modification (ICD-9-CM) format), procedures (Current Procedural Terminology, $4^{\text {th }}$ Edition (CPT-4)), retail and mail order prescription records (National Drug Code (NDC), generic name, and quantity dispensed), demographic 
information (year of birth, gender, U.S. census region), health plan, and start and stop dates for plan enrollment and pharmacy benefits. This database has been used in other epidemiologic studies of IBD (19-24), and has been reported to be representative of the commercially insured population in the US (25). Encrypted unique patient identifiers were used to integrate pharmacy and medical claims and all analyses were based on de-identified data. The study protocol was granted an exemption by the Institutional Review Board at University of North Carolina because it involved the use of existing and de-identified data.

\section{Study Population}

Case ascertainment-All individuals with at least 12 months of continuous health plan enrollment were eligible for inclusion in this analysis. Cases of Crohn's disease (CD), ulcerative colitis (UC), and indeterminate IBD were identified using a previously reported administrative definition (23). This definition included subjects with at least 3 healthcare contacts, on different days, associated with an ICD-9-CM diagnosis code for CD (555.xx) or UC (556.xx), or subjects with at least 1 claim for CD or UC and at least 1 pharmacy claim for any of the following medications: mesalamine, olsalazine, balsalazide, sulfasalazine, 6mercaptopurine, azathioprine, infliximab, adalimumab, and enteral budesonide. For subjects who had claims for both $\mathrm{CD}$ and UC, disease assignment was made according to the majority of the last 9 claims (See Figure 1).

In order to measure isotretinoin exposure preceding the diagnosis of IBD, we required that cases have a minimum of 12 months of health plan enrollment prior to the first IBD-related claim (i.e. 12 months without a diagnosis code for $\mathrm{CD}$ or $\mathrm{UC}$, or a prescription for oral or rectal aminosalicylates, 6-mercaptopurine, azathioprine, methotrexate, infliximab, or adalimumab). Isotretinoin exposure was measured during this 12 month period (exposure period).

Control ascertainment-For each case, we randomly selected up to 3 non-IBD controls without any claims for $\mathrm{CD}$ or $\mathrm{UC}$ over the duration of their health plan enrollment. Controls were matched to cases on the following factors: age (within 2 year increments), gender, U.S. census region (East, South, Midwest, and West), health plan, and length of enrollment (in 3 month increments).

\section{Assessment of isotretinoin exposure}

The number of isotretinoin prescriptions occurring in the 12 months prior to the first diagnosis of $\mathrm{CD}$ or $\mathrm{UC}$ for cases, or in the first 12 months of enrollment for controls was identified using NDC codes for generic isotretinoin and the following trade names: Accutane ${ }^{\circledR}$, Claravis ${ }^{\circledR}$, Sotret ${ }^{\circledR}$ and Amnesteem $\AA$. Both cases and controls had exposure status assessed over an equal time frame preceding ascertainment of incident disease status. In our primary analysis, we considered isotretinoin use as a dichotomous (ever/never) exposure. We subsequently performed a number of sub-analyses examining dosage and length of treatment, including 1) maximum daily dose of isotretinoin, 2) any dose-escalation during therapy, and 3) sum of the number of days supplied for all prescriptions. Daily dose was calculated as: (quantity/days supplied) $\times$ strength.

\section{Secondary analyses}

Because the diagnosis of IBD can be delayed, and because of wide variation in latency time between isotretinoin exposure and the development of IBD in published case reports, we repeated our analysis requiring a longer exposure period ( 24 months prior to the first IBD diagnosis or IBD-related prescription for cases, 24 months of continuous enrollment for controls). 
To minimize the possibility of misclassifying cases of self-limited colitis, ischemic colitis, or other non-IBD conditions were included as cases, we repeated our analyses using a more stringent case definition (> 5 claims for CD or UC, occurring on separate days).

Isotretinoin is primarily prescribed amongst younger age groups for the treatment of acne $(26,27)$, whereas IBD can affect persons of all ages (28). Because of this discrepancy, we performed an additional subanalysis restricted to individuals subjects between the ages of 12 and 45 , the peak age range of isotretinoin users (1).

Isotretinoin is occasionally prescribed for the treatment of non-acne dermatologic disorders. Some of these conditions (e.g. pyoderma faciale, pyodermatitis-pyostomatitis vegetans, and hydradenitis suppurativa) are known extra-intestinal manifestations of IBD that may precede its diagnosis $(11,29,30)$. To minimize the possibility that isotretinoin use for such conditions were biasing our results (i.e. that subclinical onset of IBD preceded isotretinoin exposure in some exposed cases), we performed an additional analysis restricting the exposure to only those with a coded diagnosis of acne vulgaris (ICD-9 code 760.1) on the same date or prior to a first prescription of isotretinoin.

In addition, to test whether the relationship between isotretinoin and IBD was confounded by acne, we evaluated whether acne was independently associated with IBD via logistic regression modeling.

\section{Sensitivity analysis}

Due to heightened awareness of teratogenicity and the associated rigorous FDA-required safety monitoring (31), persons taking isotretinoin are more likely to be scrutinized by a physician than their same age peers. This is particularly true for adolescents, who generally have low healthcare utilization in the US (32). It is possible that this might lead to differential outcome misclassification (i.e. higher sensitivity of IBD diagnosis amongst exposed persons). To evaluate the potential impact of differential outcome misclassification in isotretinoin exposed and un-exposed individuals, we performed a sensitivity analysis varying the rate of detection in the unexposed group-from 50\% to $99 \%$, while keeping the sensitivity of the exposed at $100 \%$. Previously reported equations for calculating expected results were used for this analysis (33).

\section{Statistical analysis}

Bivariate analyses for each non-matching variable were performed with Pearson's chisquared tests to compare distributions between cases and controls. To estimate the association between exposure to isotretinoin and a diagnosis of IBD, all odds ratio (OR) estimates were obtained from conditional logistic regression to account for the matching strata of age (within 2 years), gender, geographic region, health plan, and duration of enrollment (within 3 months). All statistical analyses were performed with STATA version 10.0 (College Station, TX).

\section{Results}

\section{Characteristics of study population}

Our study population contained 8,189 cases (3664 CD, 4428 UC, and 97 IBD unspecified) and 21,832 controls. Women comprised $56 \%$ of the study population. Because sex, age and geographic region were matched by design, distribution of these variables in cases and controls was the same. Isotretinoin use during the 12 month exposure period occurred in 60 individuals ( 24 cases and 36 controls). Cases and controls had similar rates of acne ( 3 and $2 \%$ respectively). Among those who were exposed to isotretinoin, 54/60 (90\%) had a 
diagnosis of acne vulgaris on the same date or preceding the prescription date (Table 1). There were no missing data for the exposure or outcome, or for the other covariates including age, gender, geographic region, acne diagnosis, or disease manifestation.

\section{Results of matched analyses}

The odds ratio for IBD in relation to any isotretinoin use was 1.68 (95\% CI $0.98,2.86$ )

(Table 2). The effect differed by type of IBD. Ulcerative colitis was strongly associated with prior isotretinoin exposure (OR 4.36, 95\% CI 1.97, 9.66), however there was no apparent association between isotretinoin exposure and Crohn's disease (OR 0.68, 95\% CI 0.28, 1.68). Amongst exposed cases, the median latency time (between first isotretinoin prescription and first IBD diagnosis) was 223 days (range 47-389).

\section{Effect of maximum daily dose, dose escalation, and duration of therapy}

Apart from analyzing isotretinoin exposure as a binary variable, further exposure analysis was performed using information on dosage and length of isotretinoin treatment. Because isotretinoin is prescribed at a range of doses, we first assessed the effect of the maximum daily dose. Compared to non-users, the odds ratio for UC for each $10 \mathrm{mg}$ increase in daily dose of isotretinoin was 1.23 (95\% CI 1.04, 1.45), while for each $20 \mathrm{mg}$ increase in daily dose, the odds ratio was 1.50 (95\% CI 1.08, 2.09). We further analyzed whether dose escalation affected risk of UC. Compared to unexposed subjects, the odds ratio for those who had their dose of isotretinoin increased at some point during the course of therapy was 15.00 (95\% CI 1.75, 128.39), while the odds ratio for those who remained on a stable dose was 2.03 (95\% CI $0.61,6.74)$. Next, we examined the effect of duration of therapy on the risk of UC. Compared to unexposed persons, the odds ratio for those taking isotretinoin for at least 60 days was 5.63 (95\% CI 2.10, 15.03), while the odds ratio for those taking isotretinoin for less than 2 months was 2.50 (95\% CI 0.61, 10.23). As shown in Table 3, the direction of the effects of maximum daily dose, dose escalation, and duration of therapy were not observed in Crohn's disease.

\section{Secondary analyses}

After increasing the length of the required exposure period to at least 24 months, the ORs for isotretinoin exposure in IBD, UC, and CD remained essentially unchanged. Similar to the 12-month exposure period analysis, isotretinoin was associated with an increased risk of UC (OR 2.90, 95\% CI 1.17, 7.20) but not CD (OR 0.89, 95\% CI 0.32, 2.52). Results of the agerestricted analysis (12-45 years of age) were similar to the results from the full study population (OR for IBD, UC, and CD of $1.55,4.90$, and 0.57 , respectively). Similarly, changing to a more stringent outcome definition requiring $\geq 5$ claims for $\mathrm{CD}$ or $\mathrm{UC}$ (in an effort to eliminate cases of self-limited colitis that may have been misclassified as IBD) did not change the effect estimates. Restricting by both age and $\geq 5$ claims generated similar results. Restricting the exposure to only subjects with a diagnosis of acne increased the adjusted OR estimates for IBD (OR 1.82, 95\% CI 1.05, 3.17) and UC (OR 5.63, 95\% CI $2.41,13.18$ ), and the association with CD remained nonsignificant (Table 4). Finally, a diagnosis of acne was not independently associated with UC (OR 1.25, 95\% CI 0.98, 1.58), CD (OR 0.96, 95\% CI 0.74, 1.25) or IBD overall (OR 1.11, 95\% CI 0.94, 1.32), making it less likely that the relationship between isotretinoin and IBD is confounded by acne.

\section{Sensitivity analysis}

We performed a sensitivity analysis to determine how differential misclassification of IBD status in isotretinoin exposed and unexposed individuals might bias our odds ratio estimates. We determined that a positive association between isotretinoin and UC could not be attributed to detection bias unless the sensitivity of UC diagnosis in the unexposed group 
was less than $50 \%$ (i.e. unless $>50 \%$ of IBD cases went undiagnosed in the unexposed group an unlikely possibility).

\section{Discussion}

In this case-control analysis of administrative claims data from a large, commerciallyinsured U.S. population including over 8000 cases of IBD, we found that prior isotretinoin exposure was associated with an increased risk of ulcerative colitis, but not Crohn's disease. There appeared to be substantial evidence of a "dose-response" relationship for ulcerative colitis. Higher doses of isotretinoin, dose escalation, and longer duration of therapy were all associated with the highest risk of UC. To our knowledge, this is the first epidemiological study to define a positive association between isotretinoin and IBD, and to address important causal criteria such as strength, temporality, specificity, and dose-related effects.

Though isolated case reports of IBD developing in isotretinoin users have been previously published, case reports and case series are unable to estimate the strength of associations and dose-response relationships. Moreover, the temporality of associations is difficult to ascertain from individual case reports (17). Bernstein et al. recently published a case-control study evaluating the association between isotretinoin and IBD using administrative claims data from Manitoba, Canada on 2,008 cases of IBD (890 UC and 1,118 CD) and 19,814 matched controls. While no association was found (OR for IBD: 1.16 (95\% CI 0.73, 1.77); UC: $1.16(95 \%$ CI $0.56,2.20)$; CD: 1.15 (95\% CI 0.61, 2.02), there are a number of methodological issues that may have led to an underestimation of the odds ratio in this study. Primarily, the median latency time (from last prescription to first diagnosis of IBD) was 906 days (range 88-2,339 days), meaning that a substantial proportion of cases had exposure to isotretinoin 3-6 years prior to diagnosis. As temporality is one of the fundamental Bradford-Hill criteria for causation (34), our study focused on exposures occurring 1 (and 2) years prior to the first IBD diagnosis. As one would expect with a temporal association, our study found a diminished effect when we included those exposures occurring within two years prior to UC diagnosis (OR: 2.90), compared to including exposures occurring only within 1 year of diagnosis (OR: 4.36). This suggests that the inclusion of exposures occurring many years prior to diagnosis in the Manitoba study may have biased the results towards the null. Furthermore, our study also analyzed the effect of dosage on the association between isotretinoin and IBD, another important causal criterion that was not evaluated in the Manitoba study. Our results suggest that if those on small doses represented a sizeable proportion of the exposed Manitoba population, this could further explain the discordant results. Finally, our study contained roughly 4 times as many IBD cases as the Canadian study, which contributes to the precision and weight of our effect estimates.

Our observation of different effects for two similar diseases, UC and CD, makes a systematic bias an unlikely explanation for the observed association. This finding is consistent with other known exposures that differentially impact the risk of one, but not both inflammatory bowel diseases including tobacco and prior appendectomy $(35,36)$. The biologic mechanism by which isotretinoin might lead to UC but not CDis unknown, , but is consistent with the understanding that ulcerative colitis and Crohn's disease have distinct immunologic features, and could be related to its effects on $\mathrm{T}$ cell function and adaptive immunity, as some have postulated (16). Moreover, the consistent finding that isotretinoin was associated with ORs $<1$ for Crohn's disease is compelling, given that retinoids are also used in the treatment of psoriasis, a disease process that is immunologically related to Crohn's. 
It should be noted that, although the relative risk for UC (estimated in this study by odds ratios) is high, the absolute risk of UC in subjects treated with isotretinoin is likely quite small. The expected number of UC cases in the exposed group (i.e. if the OR were 1.0) would be roughly 4 , meaning that 14 "excess" cases were observed within a large population $(4,428)$ of UC patients. This low absolute risk should be emphasized when interpreting these results or applying them to patient care decisions. Many patients with disfiguring acne would likely (and do regularly) accept even moderate risks of isotretinoin in order to ameliorate their condition, as this drug is uniquely efficacious, especially for severe or recalcitrant acne $(37,38)$.

Our analysis was designed to address some of the potential limitations inherent in using administrative data. First, cases were identified using diagnosis codes rather than clinical records, leading to possible outcome misclassification. To address this, we used administrative definitions that were identical or similar to those used previously by our group and others $(23,39,40)$. Previous studies have validated an administrative claims definition of a single IBD ICD-9 diagnosis code, which had a PPV of $81-84 \%$ and a sensitivity of $>90 \%(40)$. We used a more specific definition ( $\geq 3$ IBD diagnoses or $\geq 1$ IBD diagnosis +1 IBD-related prescription) in order to minimize the possibility of false positive cases. Furthermore, misclassifying unaffected individuals as cases and vice versa would underestimate the true exposure-outcome relationship, such that actual risks may be even greater than reported here.

A one year period free of claims for IBD may not have been sufficiently long to exclude all prevalent cases (e.g. cases of mild or quiescent IBD with infrequent claims). To protect against this contamination, we also excluded cases with an IBD-related prescription during the 1 year period. Although residual contamination of incident cases with prevalent cases was still possible, such misclassification would bias our results away from the null only if prevalent cases were more likely than controls to have been treated with isotretinoin, which is unlikely.

It is theoretically possible that isotretinoin is associated with self-limited colitis that was mis-coded as ulcerative colitis. To assess for this, we used a more stringent definition of UC that is unlikely to have included other forms of colitis ( $\geq 5$ coded visits on separate days), and found the strength of the association to be unchanged.

Persons exposed to isotretinoin have greater contact with the healthcare system and receive more scrutiny (due to known isotretinoin toxicity and tightly-regulated prescribing practices(31)), and hence may be more likely to have their IBD diagnosed and coded than non-isotretinoin users. We performed a sensitivity analysis to address this concern, and determined that detection bias would have to be quite strong (> 50\% reduction in detection in unexposed) in order to nullify the measured positive effect.

The only opportunity for missing data in an administrative claims database would be encounters for which a claim was not submitted. However we believe this would be a rare occurrence given the relatively high cost of both isotretinoin prescriptions and the diagnosis and management of IBD. We measured exposure by prescriptions dispensed, and do not have data regarding adherence and/or discontinuation of therapy. However, given that these are generally highly motivated patients and that in our study $82 \%$ of subjects had $\geq 2$ dispensings, we believe that isotretinoin prescribing and actual exposure are closely related. Because this study utilized an insurance claims database, our results may not be generalizable to uninsured populations, though we have no reason to suspect that the biologic effects of isotretinoin would differ based on insurance status. 
We did not assess use of antibiotics preceding exposure to isotretinoin. Prior studies have suggested either no association or a small positive association between prior antibiotic exposure and the development of IBD; however, most of the literature is related to Crohn's disease not ulcerative colitis (41-43). Therefore, we do not believe that antibiotic use is likely to be a significant confounder of the relationship between isotretinoin and UC.

Finally, it is important to note that some dermatologic conditions such as pyoderma faciale may be extra-intestinal manifestations of IBD. If isotretinoin was prescribed for such conditions in our study population, it would potentially bias our results away from the null. However, over $90 \%$ of isotretinoin users (and 100\% of exposed cases) had a diagnosis of acne vulgaris, suggesting that isotretinoin use for these rare cutaneous manifestations of IBD is uncommon. Furthermore, restricting the exposure definition to subjects with a diagnosis of acne in conjunction with isotretinoin did not alter our estimates. Though some have speculated an association between acne itself and inflammatory bowel disease (44), in our study a diagnosis of acne was not independently associated with either UC or CD, and adjustment for acne in multivariate analysis did not substantially alter our OR estimates or their statistical significance.

In conclusion, this case-control study demonstrates a possible causal association between isotretinoin use and ulcerative colitis, but not Crohn's disease. Temporality, effect specificity, and increasing effects for both intensity and duration of therapy provide further evidence of causation. As this is the first epidemiological study to describe a positive association between isotretinoin and ulcerative colitis, these results should be confirmed by additional studies in other populations. Though the absolute risk of developing IBD after taking isotretinoin is likely quite small, clinicians prescribing isotretinoin as well as prospective patients should be aware of this possible association.

\section{Acknowledgments}

Financial support: Dr. Crockett is supported by a grant from the National Institutes of Health: T32 DK 07634. The research was also supported by KL2 RR025746 (MDK) and P30 DK034987 (RSS).

\section{Abbreviations}

$\begin{array}{ll}\text { IBD } & \text { inflammatory bowel disease } \\ \text { CD } & \text { Crohn's disease } \\ \text { UC } & \text { ulcerative colitis } \\ \text { OR } & \text { odds ratio } \\ \text { 95\% CI } & 95 \% \text { Confidence interval }\end{array}$

\section{References}

1. Wysowski DK, Swann J, Vega A. Use of isotretinoin (Accutane) in the United States: rapid increase from 1992 through 2000. J Am Acad Dermatol. 2002; 46:505-9. [PubMed: 11907498]

2. Benke PJ. The isotretinoin teratogen syndrome. JAMA. 1984; 251:3267-9. [PubMed: 6587131]

3. Lammer EJ, Chen DT, Hoar RM, et al. Retinoic acid embryopathy. N Engl J Med. 1985; 313:83741. [PubMed: 3162101]

4. Rosa FW. Teratogenicity of isotretinoin. Lancet. 1983; 2:513. [PubMed: 6136666]

5. Ahmed R, Pezzone M. Isoretinoin-associated ulcerative colitis. American Journal of Gastroenterology. 2006; 101:S394.

6. Bankar RN, Dafe CO, Kohnke A, et al. Ulcerative colitis probably associated with isotretinoin. Indian J Gastroenterol. 2006; 25:171-2. [PubMed: 16877847] 
7. Borobio E, Arin A, Valcayo A, et al. Isotretinoin and ulcerous colitis. An Sist Sanit Navar. 2004; 27:241-3. [PubMed: 15381956]

8. Mennecier D, Poyet R, Thiolet C, et al. Ulcerative colitis probably induced by isotretinoin. Gastroenterologie Clinique Et Biologique. 2005; 29:1306-1307. [PubMed: 16518299]

9. Passier JL, Srivastava N, van Puijenbroek EP. Isotretinoin-induced inflammatory bowel disease. Neth J Med. 2006; 64:52-4. [PubMed: 16517990]

10. Reniers DE, Howard JM. Isotretinoin-induced inflammatory bowel disease in an adolescent. Ann Pharmacother. 2001; 35:1214-6. [PubMed: 11675849]

11. Rolanda C, Macedo G. Isotretinoin and inflammatory bowel disease. Am J Gastroenterol. 2007; 102:1330. [PubMed: 17531023]

12. Accutane package insert. [cited 2009 March 1]; Available from: http://www.rocheusa.com/products/accutane/pi.pdf

13. Voreacos, D. Roche Found Liable in First Of 400 Suits Over Accutane; Acne Drug Ruled to Cause Bowel Disease. The Washington Post; May 30. 2007

14. Roche Discontinues and Plans to Delist Accutane in the US. [cited July 25, 2009]; Available from: http://www.rocheusa.com/newsroom/current/2009/pr2009062601.html

15. Reddy D, Siegel CA, Sands BE, et al. Possible association between isotretinoin and inflammatory bowel disease. Am J Gastroenterol. 2006; 101:1569-73. [PubMed: 16863562]

16. Shale M, Kaplan GG, Panaccione R, et al. Isotretinoin and intestinal inflammation: what gastroenterologists need to know. Gut. 2009; 58:737-41. [PubMed: 19433589]

17. Crockett S, Gulati A, Sandler RS, Kappelman MD. A causal association between Accutane and IBD has yet to be established. Am J Gastroenterol. 2009 (In press).

18. Bernstein CN, Nugent Z, Longobardi T, et al. Isotretinoin Is Not Associated With Inflammatory Bowel Disease: A Population-Based Case-Control Study. Am J Gastroenterol. 2009

19. Bickston SJ, Waters HC, Dabbous O, et al. Administrative claims analysis of all-cause annual costs of care and resource utilization by age category for ulcerative colitis patients. J Manag Care Pharm. 2008; 14:352-62. [PubMed: 18500913]

20. Cohen RD, Waters HC, Tang B, et al. Effects of fistula on healthcare costs and utilization for patients with Crohn's disease treated in a managed care environment. Inflamm Bowel Dis. 2008; 14:1707-14. [PubMed: 18618630]

21. Ollendorf DA, Lidsky L. Infliximab drug and infusion costs among patients with Crohn's disease in a commercially-insured setting. Am J Ther. 2006; 13:502-6. [PubMed: 17122530]

22. Long MD, Porter CQ, Sandler RS, et al. Suboptimal rates of cervical testing among women with inflammatory bowel disease. Clin Gastroenterol Hepatol. 2009; 7:549-53. [PubMed: 18996498]

23. Kappelman MD, Rifas-Shiman SL, Kleinman K, et al. The prevalence and geographic distribution of Crohn's disease and ulcerative colitis in the United States. Clin Gastroenterol Hepatol. 2007; 5:1424-9. [PubMed: 17904915]

24. Kappelman MD, Rifas-Shiman SL, Porter CQ, et al. Direct health care costs of Crohn's disease and ulcerative colitis in US children and adults. Gastroenterology. 2008; 135:1907-13. [PubMed: 18854185]

25. Stempel DA, Mauskopf J, McLaughlin T, et al. Comparison of asthma costs in patients starting fluticasone propionate compared to patients starting montelukast. Respir Med. 2001; 95:227-34. [PubMed: 11266241]

26. Azoulay L, Oraichi D, Berard A. Patterns and utilization of isotretinoin for acne from 1984 to 2003: is there need for concern? Eur J Clin Pharmacol. 2006; 62:667-74. [PubMed: 16791584]

27. Stern RS. Medication and medical service utilization for acne 1995-1998. J Am Acad Dermatol. 2000; 43:1042-8. [PubMed: 11100021]

28. Herrinton LJ, Liu L, Lewis JD, et al. Incidence and prevalence of inflammatory bowel disease in a Northern California managed care organization, 1996-2002. Am J Gastroenterol. 2008; 103:19982006. [PubMed: 18796097]

29. Dessoukey MW, Omar MF, Dayem HA. Pyoderma faciale: manifestation of inflammatory bowel disease. Int J Dermatol. 1996; 35:724-6. [PubMed: 8891823] 
30. Brinkmeier T, Frosch PJ. Pyodermatitis-pyostomatitis vegetans: a clinical course of two decades with response to cyclosporine and low-dose prednisolone. Acta Derm Venereol. 2001; 81:134-6. [PubMed: 11501652]

31. Mitchell AA, Van Bennekom CM, Louik C. A pregnancy-prevention program in women of childbearing age receiving isotretinoin. N Engl J Med. 1995; 333:101-6. [PubMed: 7777014]

32. Ma J, Wang Y, Stafford RS. U.S. adolescents receive suboptimal preventive counseling during ambulatory care. J Adolesc Health. 2005; 36:441. [PubMed: 15841517]

33. Greenland, SLT. Bias Analysis. In: Rothman, KGS.; Lash, TL., editors. Modern Epidemiology. 3. Philadelphia, PA: Lippincott Williams \& Wilkins; 2008. p. 358-359.

34. Hill AB. The Environment and Disease: Association or Causation? Proc R Soc Med. 1965; 58:295-300. [PubMed: 14283879]

35. Andersson RE, Olaison G, Tysk C, et al. Appendectomy and protection against ulcerative colitis. $\mathrm{N}$ Engl J Med. 2001; 344:808-14. [PubMed: 11248156]

36. Hanauer SB. Inflammatory bowel disease: epidemiology, pathogenesis, and therapeutic opportunities. Inflamm Bowel Dis. 2006; 12 (Suppl 1):S3-9. [PubMed: 16378007]

37. Amichai B, Shemer A, Grunwald MH. Low-dose isotretinoin in the treatment of acne vulgaris. J Am Acad Dermatol. 2006; 54:644-6. [PubMed: 16546586]

38. Jones H, Blanc D, Cunliffe WJ. 13-cis retinoic acid and acne. Lancet. 1980; 2:1048-9. [PubMed: 6107678]

39. Bernstein CN, Wajda A, Svenson LW, et al. The epidemiology of inflammatory bowel disease in Canada: a population-based study. Am J Gastroenterol. 2006; 101:1559-68. [PubMed: 16863561]

40. Herrinton LJ, Liu L, Lafata JE, et al. Estimation of the period prevalence of inflammatory bowel disease among nine health plans using computerized diagnoses and outpatient pharmacy dispensings. Inflamm Bowel Dis. 2007; 13:451-61. [PubMed: 17219403]

41. Card T, Logan RF, Rodrigues LC, et al. Antibiotic use and the development of Crohn's disease. Gut. 2004; 53:246-50. [PubMed: 14724158]

42. Hildebrand H, Malmborg P, Askling J, et al. Early-life exposures associated with antibiotic use and risk of subsequent Crohn's disease. Scand J Gastroenterol. 2008; 43:961-6. [PubMed: 19086166]

43. Gilat T, Hacohen D, Lilos P, et al. Childhood factors in ulcerative colitis and Crohn's disease. An international cooperative study. Scand J Gastroenterol. 1987; 22:1009-24. [PubMed: 3685876]

44. Murphy CL, Gibson D, Meyers LS. Inflammatory bowel disease and acne. Am J Gastroenterol. 2009; 104:2370. [PubMed: 19727108] 


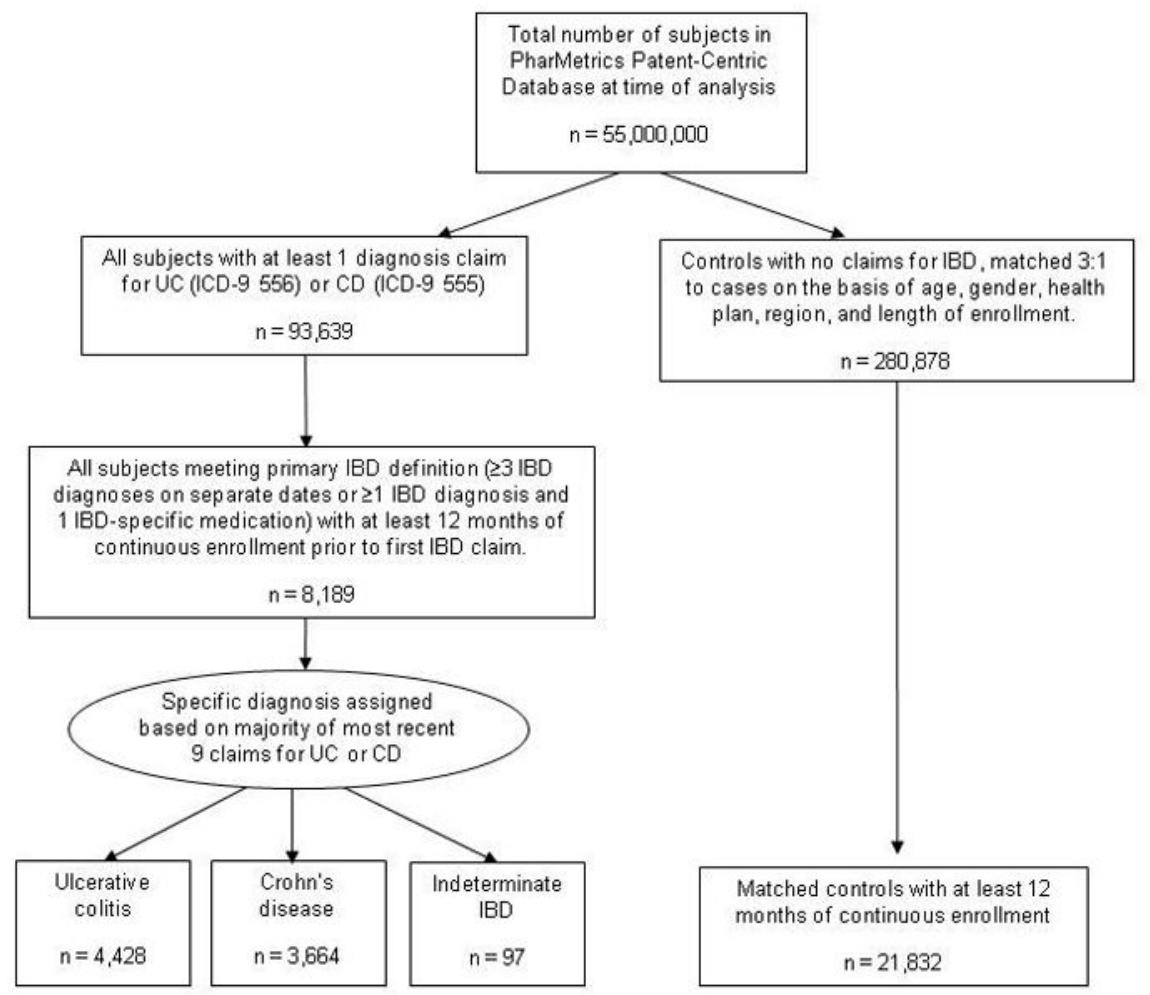

Figure 1.

Derivation of study population including subjects with inflammatory bowel disease and matched controls

UC: ulcerative colitis; CD: Crohn's disease; IBD: inflammatory bowel disease; ICD-9: International Classification of Diseases, $9^{\text {th }}$ Revision code 


\section{TABLE 1}

Characteristics of the study population*

\begin{tabular}{|c|c|c|}
\hline & $\begin{array}{c}\text { Cases }(\mathbf{n}=\mathbf{8 , 1 8 9}) \\
\mathbf{n}(\%)\end{array}$ & $\begin{array}{c}\text { Controls }(n=21,832) \\
n(\%)\end{array}$ \\
\hline \multicolumn{3}{|l|}{ Isotretinoin ${ }^{\dagger}$} \\
\hline Yes & $24(0)$ & $36(0)$ \\
\hline No & $8,165(100)$ & $21,796(100)$ \\
\hline \multicolumn{3}{|l|}{ Gender } \\
\hline Female & $4,545(56)$ & $12,147(56)$ \\
\hline Male & $3,644(44)$ & $9,685(44)$ \\
\hline \multicolumn{3}{|l|}{ Age (years) } \\
\hline$<10$ & $61(1)$ & $331(2)$ \\
\hline $10-19$ & $731(9)$ & $2,239(10)$ \\
\hline $20-29$ & $1,031(13)$ & 2,747 (13) \\
\hline $30-39$ & $1,541(19)$ & $4,397(20)$ \\
\hline $40-49$ & $2,068(25)$ & $5,677(26)$ \\
\hline $50-59$ & $2,041(25)$ & $5,140(24)$ \\
\hline$\geq 60$ & $716(9)$ & $1,301(6)$ \\
\hline \multicolumn{3}{|l|}{ Region ${ }^{\ddagger}$} \\
\hline East & $1,594(18)$ & $3,813(17)$ \\
\hline South & $7,233(24)$ & $5,301(24)$ \\
\hline West & $6,488(22)$ & $4,738(22)$ \\
\hline Midwest & $10,893(36)$ & $7,980(37)$ \\
\hline \multicolumn{3}{|l|}{ Acne diagnosis $\S$} \\
\hline Yes & $217(3)$ & $502(2)$ \\
\hline No & $7,972(97)$ & $21,330(98)$ \\
\hline \multicolumn{3}{|l|}{ Disease manifestation } \\
\hline Crohn's disease & $3,664(45)$ & $\mathrm{n} / \mathrm{a}$ \\
\hline Ulcerative colitis & $4,428(54)$ & \\
\hline Indeterminate IBD & $97(1)$ & \\
\hline
\end{tabular}

* Cases were persons with Crohn's disease, ulcerative colitis, or indeterminate inflammatory bowel disease. Controls were matched in a 3:1 ratio by age, gender, region, health plan, and length of plan enrollment.

${ }^{\dagger}$ At least 1 prescription for isotretinoin in the 1 year preceding IBD diagnosis (cases) or first 1 year of enrollment (controls).

${ }^{*}$ Region as defined by the US census boundaries.

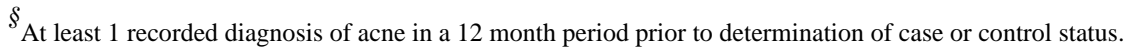

IBD: inflammatory bowel disease 
TABLE 2

Odds Ratios (95\% CI) for Inflammatory Bowel Disease, Crohn's Disease and Ulcerative Colitis by exposure to isotretinoin.*

\begin{tabular}{|c|c|c|c|}
\hline & $\mathbf{I B D}^{\dagger}$ & UC & CD \\
\hline Exposure & OR $(95 \%$ CI $)$ & OR $(95 \%$ CI $)$ & OR $(95 \%$ CI $)$ \\
\hline Isotretinoin use & $1.68(0.98,2.86)$ & $4.36(1.97,9.66)$ & $0.68(0.28,1.68)$ \\
\hline
\end{tabular}




\section{TABLE 3}

Daily dose, dose escalation, and days supplied of isotretinoin and odds ratios of inflammatory bowel disease, ulcerative colitis, and Crohn's disease.*

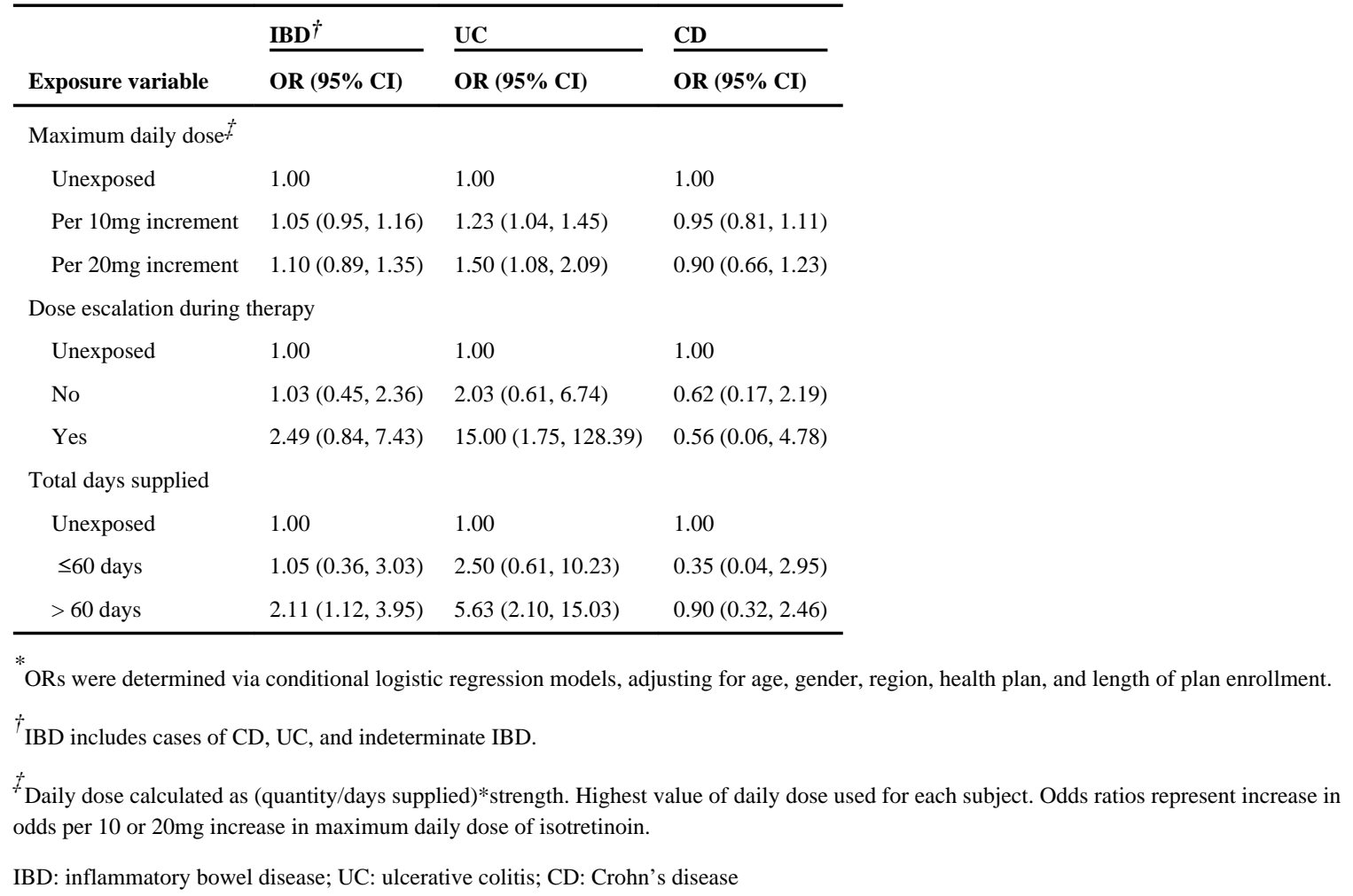


TABLE 4

Odds ratios for inflammatory bowel disease, Crohn's disease and ulcerative colitis by exposure to isotretinoin in secondary analyses.*

\begin{tabular}{|c|c|c|c|}
\hline & $\underline{\mathbf{I B D}^{\dagger}}$ & $\underline{\mathbf{U C}}$ & CD \\
\hline Secondary analyses & OR $(95 \% \mathrm{CI})$ & OR $(95 \% \mathrm{CI})$ & OR $(95 \% \mathrm{CI})$ \\
\hline 2 year minimum & $1.67(0.87,3.23)$ & $2.90(1.17,7.20)$ & $0.89(0.32,2.52)$ \\
\hline Age restricted ${ }^{\S}$ & $1.55(0.88,2.73)$ & $4.90(2.05,11.69)$ & $0.57(0.22,1.49)$ \\
\hline$\geq 5$ diagnoses ${ }^{\square}$ & $1.76(0.95,3.25)$ & $4.19(1.61,10.89)$ & $0.90(0.35,2.27)$ \\
\hline$\geq 5$ diagnoses \& age restricted & $1.76(0.93,3.30)$ & $5.14(1.90,13.93)$ & $0.74(0.27,2.01)$ \\
\hline Acne only I & $1.82(1.05,3.17)$ & $5.63(2.41,13.18)$ & $0.62(0.23,1.63)$ \\
\hline
\end{tabular}

ORs were determined via conditional logistic regression models, adjusting for age, gender, region, health plan, and length of plan enrollment.

${ }^{\dagger}$ IBD includes cases of CD, UC, and indeterminate IBD.

${ }^{*}$ Results after lengthening the potential exposure period to 24 months prior to IBD diagnosis (or first 24 months of health plan enrollment for controls).

$\S_{\text {Results after restricting age of cases and controls to peak age of isotretinoin use: } 12-45 \text { years }}$

${ }^{\square}$ Results after using a more stringent definition for IBD cases, requiring $\geq 5$ diagnosis codes on separate dates

II Exposure limited to persons who had a diagnosis of acne coded on the same visit or prior to a prescription for isotretinoin

IBD: inflammatory bowel disease; UC: ulcerative colitis; CD: Crohn's disease 